\title{
Targeting stem cell niche can protect hematopoietic stem cells from chemotherapy and G-CSF treatment
}

\author{
Sidan Li ${ }^{1,2}$, Dehui Zou' ${ }^{1}$, Changhong Li ${ }^{1}$, Hengxing Meng ${ }^{1}$, Weiwei Sui ${ }^{1}$, Sizhou Feng ${ }^{1}$, Tao Cheng ${ }^{1}$,
} Qiongli Zhai ${ }^{3^{*}}$ and Lugui Qiu ${ }^{1^{*}}$

\begin{abstract}
Introduction: Hematopoietic stem/progenitor cells (HSPCs) reside in a tightly controlled local microenvironment called bone marrow niche. The specialized microenvironment or niche not only provides a favorable habitat for HSPC maintenance and development but also governs stem cell function.

Method: We investigated the effect of cytotoxic drugs on bone marrow niche. To mimic the multiple rounds of chemotherapy followed by autologous hematopoietic stem cells (HSCs) transplantation in a clinical setting, we further verified the hypothesis that targeting the niche might improve stem cell-based therapies in mouse models.

Results: We found that multiple rounds of cytotoxic drug treatment significantly disrupted niche and serum osteocalcin level was significantly reduced after treatment in autologous HSPCs transplanted patients $(P=0.01)$. In mouse models, the number of $\mathrm{CD}^{-} 5^{-} \mathrm{Te} 119^{-} \mathrm{OPN}^{+}$osteoblasts was significantly reduced after multiple rounds of chemotherapies and granulocyte colony stimulating factor (G-CSF) treatment $(P<0.01)$. Parathyroid hormone (PTH) or receptor activator of nuclear factor kappa-B ligand (RANKL) treatment significantly increased the number of HSCS mobilized into peripheral blood (PB) for stem cell harvesting and protected stem cells from repeated exposure to cytotoxic chemotherapy. Treatments with G-CSF and PTH significantly increased the preservation of the HSC pool $(P<0.05)$. Moreover, recipient mice transplanted with circulation HSPCs that were previously treated with PTH and RANKL showed robust myeloid and lymphatic cell engraftment compared to the mice transplanted with HSCs after chemotherapy or G-CSF treatment.
\end{abstract}

Conclusion: These data provide new evidence that the niche may be an important target for drug-based stem cell therapy.

\section{Introduction}

Hematopoietic stem cell transplantation (SCT) has provided lifesaving treatment for many hematological disorders, but a significant proportion of patients who are eligible for autologous SCT fail to mobilize a sufficient number of $\mathrm{CD}^{+} 4^{+}$hematopoietic stem/progenitor cells (HSPCs), which is called "poor mobilization", owing to various premobilization (predictive) factors such as prior

\footnotetext{
*Correspondence: qionglizhai@hotmail.com; drqiu99@medmail.com.cn ${ }^{3}$ Department of Pathology, Tianjin Medical University Cancer Institute and Hospital, National Clinical Research Center for Cancer, Key Laboratory of Cancer Prevention and Therapy, Tianjin 300060, China

'State Key Laboratory of Experimental Hematology, Institute of Hematology and Hospital of Blood Diseases, Chinese Academy of Medical Sciences and Peking Union of Medical College, 288 Nanjing Road, Tianjin 30020, China Full list of author information is available at the end of the article
}

treatment with stem cell toxic drugs, underlying disease, age, prior radiotherapy, and bone marrow involvement [1-3]. Poor mobilization has disastrous consequences for patients, with potential loss of transplant as a treatment option. Moreover, 5-10 \% of healthy donors cannot obtain adequate HSPCs for allogenetic transplantation after granulocyte colony-stimulating factor (G-CSF) treatment $[1,4]$. Repeated attempts during the mobilization process will increase resource use, but morbidity and patient/donor inconvenience are also increased in the meantime. How to improve the mobilization efficiency is therefore becoming a challenging topic for hematological scholars $[5,6]$.

Poor mobilization may result from significant depletion of the bone marrow hematopoietic stem cell (HSC) pool caused by G-CSF. Since the specialized microenvironment 
(niche) governs stem cell function [7, 8], targeting the stem cell niche may change the fate of stem cells. Our previous studies demonstrated that, except for the proteolytic enzymes, cellular components of osteoblasts and osteoclasts are closely related to G-CSF-induced HSPC mobilization in healthy donors $[9,10]$. Recognition of the intimate relationship between endosteal niche cells (osteoblasts and osteoclasts) and HSPCs affords the possibility of targeting the niche to improve stem cell mobilization efficiency. The role of parathyroid hormone (PTH) in activating osteoblasts triggered researchers to investigate the possible effect of PTH on HSPCs. The pharmacological role of PTH in HPSCs during G-CSF-induced mobilization has been confirmed in a phase I clinical trial [11]. Moreover, it has been found that the resorption of osteoclasts stimulated by receptor activator of nuclear factor kappa-B ligand (RANKL) can reduce the level of stem cell niche components along the endosteum and finally trigger HSPC mobilization, so RANKL may be used together with other mobilization agents in clinical HSPC transplantation protocols [12]. In our previous study, PTH/RANKL was added to manipulatively interrupt the bone remodeling balance and then increase the number of HSPCs mobilized into the peripheral blood (PB). We demonstrated that the imbalance of bone remodeling can facilitate HSPC mobilization [9], and targeting the HSC niche may improve mobilization efficiency.

In this study, the role of bone remodeling in G-CSFinduced mobilization was examined in clinical specimens from autograft patients, and several animal models mimicking clinical mobilization situations were established to explore the possibility of improving poor mobilization.

\section{Materials and methods Sample collection}

PB samples from 10 autograft patients (including three non-Hodgkin's lymphoma (NHL) cases, two myeloma cases, and five cases with acute lymphoblastic leukemia (ALL)) were first collected after diagnosis (before treatment). A median of four chemotherapy cycles (range 3-6 cycles) was then administered to these patients [13-15]. Before the mobilization course, PB samples were again collected from patients (steady state). All patients were autografted in the first remission. The mobilization course consisted of subcutaneous injection of human recombinant G-CSF (5 $\mu \mathrm{g} / \mathrm{kg} /$ day, twice a day, Filgrastim; Japan) used in the recovery phase of myelotoxic chemotherapy (single-agent cyclophosphamide (China) infusion or mitoxantrone (China) plus cytarabine (China)). Serum samples were collected by centrifugation at $500 \times g$ for 10 minutes and stored at $-80{ }^{\circ} \mathrm{C}$ for assay. Human samples were used in accordance with approved procedures by the Human Experimentation and Ethics Committee of the Institute of Hematology and Blood Diseases Hospital,
Chinese Academy of Medical Sciences (CAMS) and Peking Union of Medical College (PUMC). We obtained consent from all patients involved in the study, including consent to participate in the study where appropriate.

\section{In vivo experiment}

C57Bl/6 and B6.SJL female mice (6-8 weeks old) were obtained from the Institute of Laboratory Animal Science, CAMS and PUMC. All of the animal handling and experimental procedures were approved by the Animal Care and Use Committee of CAMS and PUMC. Mice were housed in sterilized micro-isolator cages and received autoclaved food and water. To study the changes of niche cells and HSPCs after cytotoxic drug treatment, C57Bl/6 (CD 45.2) mice were injected intraperitoneally with cyclophosphamide (CTX) (Sigma, Sigma-Aldrich, St Louis, MO, USA) at a dose of $5 \mathrm{mg}$ once every 2 weeks for a total of four cycles, and then injected intraperitoneally with saline (CTLs group) or recombinant human G-CSF (Filgrastim) (Gs group) at a dose of 250 $\mu \mathrm{g} / \mathrm{kg} /$ day for 8 consecutive days. At the end of 10 weeks (day 71, 8-week treatment period and 2-week recovery period), mice were killed and the functions of osteoblast and HSPCs were tested (Fig. 1).

To imitate the settings of autologous transplantation following chemotherapy, after each of the four cyclophosphamide treatments already mentioned C57B1/6 mice were injected intraperitoneally with either saline (group CTL) or $250 \mu \mathrm{g} / \mathrm{kg} /$ day G-CSF (Filgrastim, groups G, PTH, P + $\mathrm{G}, \mathrm{P}+\mathrm{R}$, and $\mathrm{P}+\mathrm{R}+\mathrm{G}$ ) for 8 consecutive days. After 8week treatments, mice were treated with G-CSF for 4 days (Filgrastim, $250 \mu \mathrm{g} / \mathrm{kg} /$ day, intraperitoneally, groups CTL and G), or rat PTH for 14 days $(80 \mu \mathrm{g} / \mathrm{kg} /$ day, intraperitoneally, group PTH; Bachem, Torrance, CA), or a combination of PTH for 14 days and G-CSF for 4 days (group P + G), or a combination of PTH for 14 days and RANKL for 5 days $(6 \mu \mathrm{g} /$ day, subcutaneous injection via the femur, group P + R; PeproTech, Rocky Hill, NJ), or a combination of PTH for 14 days, RANKL for 5 days, and G-CSF for 4 days (group $P+R+G$ ). At the end of treatments, mice were sacrificed, and bone marrow HSPCs (groups CTL, G, and PTH) and HSPCs mobilized into the blood (groups CTL, G, P + G, P + R, and P + R + G) were assessed by competitive repopulation assay (CRA) (shown in Fig. 1).

\section{Isolation of bone marrow nuclear cells and osteoblasts}

Total bone marrow nuclear cells were isolated from mouse femurs by flushing with phosphate-buffered saline (PBS) plus $2 \%$ fetal bovine serum. Red blood cells and debris were removed by ammonium-chloride-potassium (ACK) lysis $\left(0.15 \mathrm{M} \mathrm{NH}_{4} \mathrm{Cl}, 1.0 \mathrm{mM} \mathrm{KHCO} 3,0.1 \mathrm{mM}\right.$ ethylenediamine tetraacetic acid (EDTA), $\mathrm{pH} 7.4$ ) and filtering through nylon mesh. Osteoblasts were isolated 


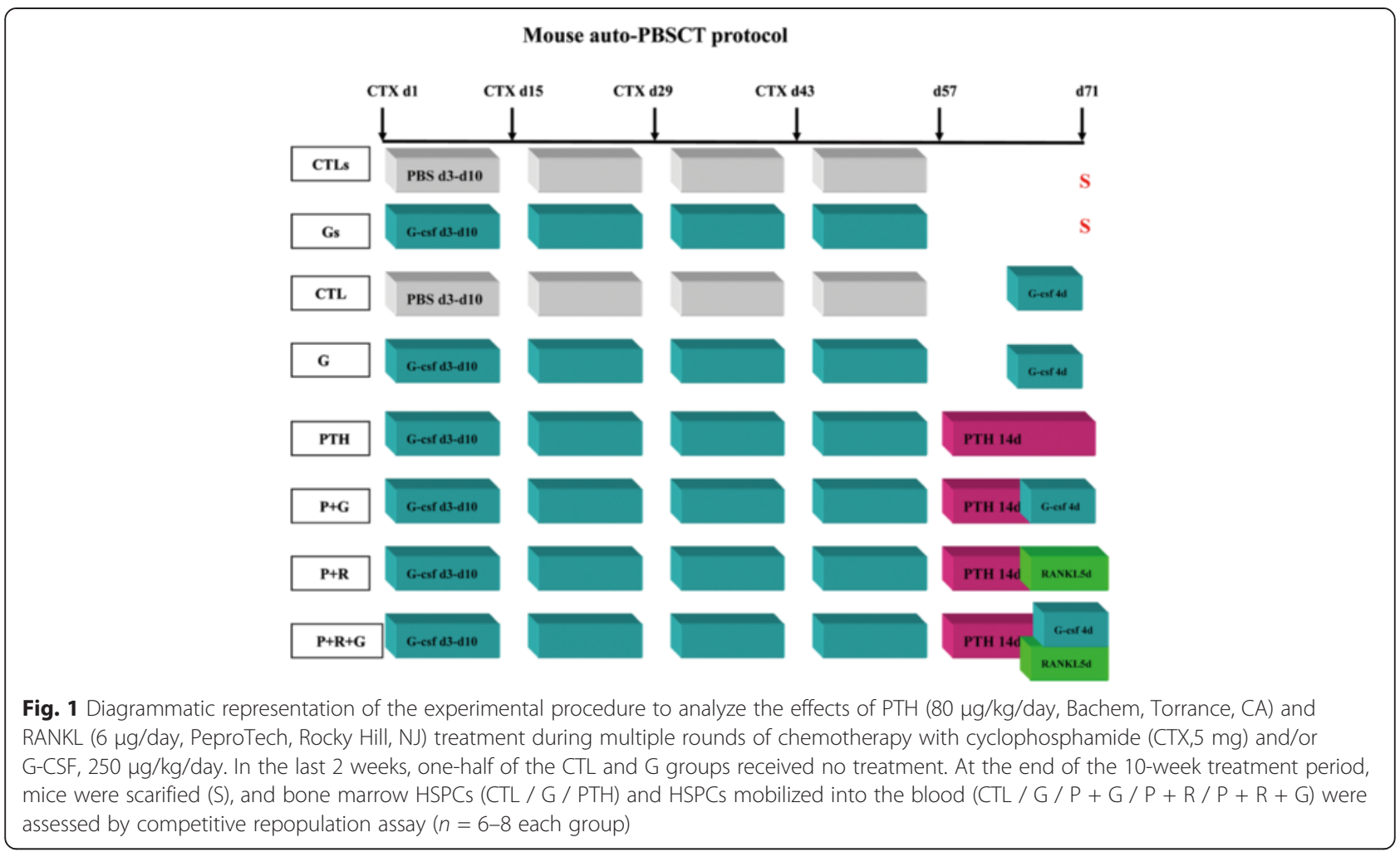

from marrow-depleted femurs by mechanical disruption (crushing with mortar and pestle), infused with PBS containing $50 \mathrm{mg} / \mathrm{ml}$ type II collagenase (GIBCO, NY) and incubated at $37{ }^{\circ} \mathrm{C}$ for 15 minutes. The collagenasetreated femurs were flushed with PBS and a similar procedure was repeated six times. Osteoblasts were collected by centrifugation at $400 \times g$ for 5 minutes and cells were pooled [16].

\section{Measurements of osteocalcin and tartrate-resistant acid phosphatase $5 \mathrm{~b}$ levels}

Serum levels of osteocalcin and tartrate-resistant acid phosphatase 5b (TRACP $5 \mathrm{~b}$ ) from mouse model and healthy donors samples were tested using immunoassay kits for osteocalcin (Biomedical Technologies, Stoughton, MA, USA for mouse; Immunodiagnostic Systems Limited, Boldon, UK for human) and TRACP 5b (Immunodiagnostic Systems Limited for mouse) following the manufacturer's instructions. The concentrations of osteocalcin and TRACP $5 \mathrm{~b}$ in each sample were calculated based on the average of different dilutions and the experiments were repeated three times.

\section{Competitive repopulation assay}

For qualitative measurement of the HSC frequency in the peripheral circulation, $300 \mu \mathrm{l} \mathrm{PB}$ were collected from the retro-orbital vein of the $\mathrm{C} 57 \mathrm{Bl} / 6$ (CD 45.2) mouse. $\mathrm{PB}$ was collected into microtainer tubes containing lithium heparin. The red cells were lysed with ACK lysing buffer and the mononuclear cells were mixed with $2.5 \times 10^{5}$ bone marrow mononuclear cells from two B6.SJL (CD 45.1) mice. To measure the bone marrow HSC cell frequency, the mice were killed with carbon dioxide and the bone marrow mononuclear cells were isolated by flushing the bone marrow cavity with PBS plus $2 \%$ fetal bovine serum. Then $2.5 \times 10^{5}$ bone marrow mononuclear cells from $\mathrm{C} 57 \mathrm{Bl} / 6$ mice were mixed with an equal number of bone marrow mononuclear cells from two B6.SJL competitor mice. These two kinds of hybrid cells were then injected into recipient B6.SJL mice that were lethally irradiated for 24 hours with 9.5 Gy radiation. The relative contribution of engraftment from the different cell sources was assessed by the detection of CD45.2 antigens in both the myeloid (defined as Side Scatter ${ }^{\text {hi }} \mathrm{Mac}_{-} 1^{+}$) and lymphoid (defined as Side Scatter ${ }^{\text {lo }} \mathrm{CD}^{+} / \mathrm{B}^{2} 20^{+}$) fraction of cells after 16 weeks. The cells were diluted and incubated with phycoerythrin (PE)-conjugated CD45.2, fluorescein isothiocyanate (FITC)-conjugated CD3, allophycocyanin (APC)-conjugated B220, and PE-Cy5-conjugated Mac-1 antibodies (eBiosciences, San Diego, CA, USA). After incubation with these antibodies, the samples were fixed and red cells were removed using BD fluorescenceactivated cell sorting (FACS) lysis solution (BD Biosciences, San Jose, CA, USA). 


\section{Flow cytometry}

To quantify osteoblast lineage cells, bone-associated cells obtained from enzymatic treatment were stained with APC-conjugated anti-mouse CD45 and Ter119 antibodies (eBiosciences, San Diego, CA, USA), and goat anti-mouse osteopontin, followed by FITC-conjugate donkey anti-goat IgG (Santa Cruz Biotechnology, Inc., Texas, USA). CD45 $-/$ Ter119 ${ }^{-} / \mathrm{OPN}^{+}$cells were enumerated [17]. The population of different cells was assessed by FACS Calibur flow cytometer and analyzed with Cell Quest software (BectonDickinson).

\section{Colony formation assay}

Nucleated bone marrow cells $\left(1.0 \times 10^{4}\right)$ were planted in $2.5 \mathrm{ml}$ methylcellulose media supplemented with a cocktail of recombinant cytokines (MethoCult 3434; StemCell Technologies, Vancouver, BC, Canada). Cultures were plated in duplicate and cultured in a humidified chamber with $5 \%$ carbon dioxide at $37{ }^{\circ} \mathrm{C}$. Colonies with at least 50 cells were counted on day 12 of culture.

\section{Reverse transcription and real-time quantitative PCR}

Mouse marrow-depleted bones were flushed with a total of $1 \mathrm{ml}$ TRIzol reagent (Invitrogen, Carlsbad, CA, USA) followed by crushing of the remaining bone in TRIzol. Reverse transcription was carried out using the Superscript First-Strand Synthesis System (Invitrogen) following the manufacturer's instructions. Real-time quantitative PCR for osteocalcin (forward primer, 5'-TCTCTCTGCTCA CTCTGCTGGCC-3'; reverse primer, 5'-TTTGTCAG ACTCAGGGCCGC-3') expression was performed on the ABI 7500 Sequence Detection System (Applied Biosystems, Foster, CA, USA). The $20 \mu \mathrm{l}$ PCR mixture consisted of $10 \mu \mathrm{l}$ Power SYBR ${ }^{\circ}$ Green PCR Master mix (Applied Biosystems), $0.5 \mu \mathrm{l}$ each primer $(100 \mu \mathrm{M}), 1 \mu \mathrm{l}$ cDNA (40 ng RNA), and $8 \mu \mathrm{lddH_{2 }}$ O. The reaction was carried out $95^{\circ} \mathrm{C}$ for 15 minutes, followed by 40 cycles of $95^{\circ} \mathrm{C}$ for 15 seconds and $60{ }^{\circ} \mathrm{C}$ for 1 minute. $\Delta \Delta \mathrm{CT}$ values were calculated from the differences between the targeted genes and internal control $\beta$-actin. Each experiment was repeated three times and the mean was calculated.

\section{Immunohistochemistry}

To mark osteoblasts, immunohistochemical staining of osteocalcin was performed on formalin-fixed, paraffinembedded sections of human biopsy specimen samples. Immunohistochemistry was carried out following the standard protocols. After dewaxing and antigen retrieval, the sections were blocked with goat serum for 1 hour and incubated with mouse anti-osteocalcin monoclonal antibody (1:100, ab13418; Abcam, Cambridge, UK) overnight at $4{ }^{\circ} \mathrm{C}$. The sections were stained with streptavidinperoxidase method and a 3,3'-diaminobenzidine (DAB, Venata Medical Systems, Basal, Switzerland) kit.
The osteoblast enumeration was performed in the growth region of all trabecular bones, but not any cortical bone (using 20x objective). Two pathologists counted the positive cells in 15 fields $(400 \times)$ per section in a blind fashion. The number of osteoblasts was averaged and signified as the osteoblast number per bone surface.

\section{Hematoxylin and eosin and TRACP staining}

Mouse femurs were fixed in $4 \%$ formaldehyde in PBS for 48 hours, decalcified in $10 \%$ EDTA ( $\mathrm{pH} 7.5$ ) for 14 days, and embedded in paraffin. Sections $(4 \mu \mathrm{m})$ were deparaffinized, rehydrated, and stained with hematoxylin and eosin (H\&E) and TRACP immunocytochemistry kit (Sigma-Aldrich Ltd, Dorset, UK) according to the manufacturer's instructions for histochemical examination $[18,19]$.

\section{Statistical analysis}

The statistical significance of overall differences among multiple groups was analyzed by the ordinary analysis of variance using SPSS 15.0 (IBM, Chicago, IL, USA). Data are presented as the mean \pm standard error of the mean. Data were analyzed using the nonparametric MannWhitney test as appropriate for the data set. $P<0.05$ was considered statistically significant.

\section{Results}

Multiple treatments of cytotoxic drugs destroy bone marrow niche osteoblasts in autologous transplantation patients

Based on our previous results, a mobilization protocol with G-CSF suppresses osteoblast function [9]. In this study, we further investigated the effect of multiple treatments of cytotoxic drugs on niche cells. PB samples from 10 patients were collected after diagnosis (before treatment) and before the mobilization chemotherapy course (steady state). We found that the osteocalcin level in the serum was significantly reduced after treatment $(22.19 \pm 1.08 \mathrm{ng} / \mathrm{ml}$ before treatment vs. $16.08 \pm 2.12$ $\mathrm{ng} / \mathrm{ml}$ steady state, $P=0.01$ ) (Fig. $2 \mathrm{a}$ ). Moreover, the number of mature osteoblasts was significantly decreased after multiple chemotherapy cycles, which is defined by osteocalcin-positive endosteum cells (Fig. 2b, c). The number of osteoblasts per bone surface was decreased from $18.55 \pm 0.32$ (before treatment) to $12.27 \pm$ 0.66 (steady state) $(P<0.05)$. These data indicate that cytotoxic drugs not only decrease the number of osteoblasts, but also suppress the activity of osteoblasts.

\section{Multiple treatments of cytotoxic drugs influence both osteoblasts and HSPCs in a mouse model}

To verify the results from patients, two mouse models (CTLs and Gs groups) were established by cyclophosphamide treatments and consecutive stimulations with G-CSF 

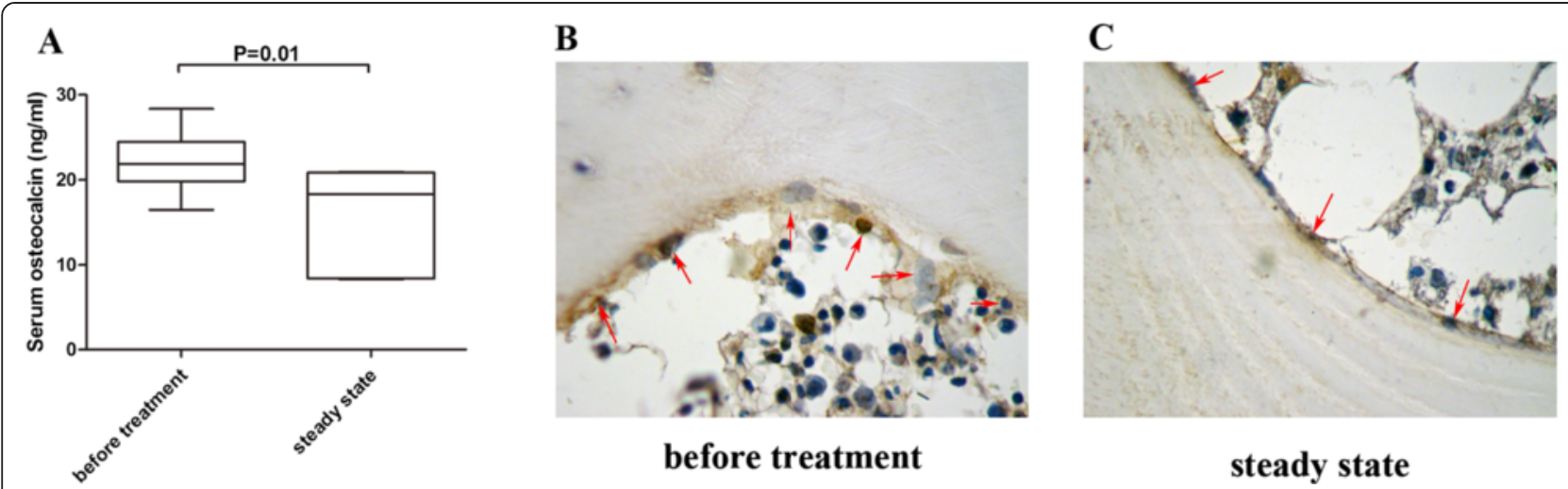

Fig. 2 Chemotherapy destroys bone marrow niche osteoblasts in autologous transplantation patients. a Serum osteocalcin concentrations decrease after multiple rounds of cytotoxic drug treatment in human. Serum samples were collected from 10 patients and their serum osteocalcin levels were measured by enzyme-linked immunosorbent assay (ELISA). b, c Representative photomicrographs of human endosteal osteoblasts after immunohistochemical staining. Osteocalcin-positive mature osteoblasts (red arrows) before treatment (b) were considerably reduced at steady state $(\mathbf{c})$ of G-CSF treatment

to mimic the patients who received high-dose chemotherapy after autologous PB SCT. Compared with untreated mice, osteocalcin mRNA expression was reduced $9.32 \pm$ 0.3 -fold in the CTLs group $(P<0.01)$ and $16.82 \pm 0.8$-fold in thex Gs group $(P<0.01)$ (Fig. 3a). The number of $\mathrm{CD}_{4} 5^{-}$Ter119 ${ }^{-} \mathrm{OPN}^{+}$osteoblasts $\left(\% \mathrm{CD} 45^{-}\right.$Ter $119^{-} \mathrm{OPN}^{+} \times$ total bone cells per femur) was significantly reduced after cyclophosphamide treatments and consecutive stimulations with G-CSF (untreated, $3993 \pm 129$ cells/femur; CTLs, $1937 \pm 196$ cells/femur; Gs, $1055 \pm 43$ cells/femur; $P<0.01$ ) (Fig. 3b, c). Moreover, the osteoblastic activity decreased to a low level. Circulation osteocalcin was decreased in the CTLs group $(33.81 \pm 1.99 \mathrm{ng} / \mathrm{ml})$ and the Gs group $(27.18 \pm 1.09 \mathrm{ng} / \mathrm{ml})$ compared with untreated mice $(59.44 \pm 3.16 \mathrm{ng} / \mathrm{ml})(P<0.01$; Fig. $3 \mathrm{~d})$. In addition, the decreased number of trabeculae was observed in the long bones from the mice in both the CTLs and Gs groups (Fig. 3e). Furthermore, compared with untreated mice $(29.17 \pm 1.22 \mathrm{U})$, the number of HSPCs in the bone marrow was significantly reduced in the CTL (21.16 \pm $1.35 \mathrm{U})$ and $\mathrm{Gs}(13.00 \pm 1.71 \mathrm{U})$ groups $(P=0.01)$ (Fig. $3 f)$. These results indicate that chemotherapy, especially in combination with G-CSF, can destroy the osteoblastic niche and lead to a significant depletion of the bone marrow HSPC pool, which may be the primary cause for poor mobilization.

\section{PTH and RANKL can efficiently activate the functions of} osteoblasts and osteoclasts during cytotoxic chemotherapy In this study, we found niche cells (osteoblasts and osteoclasts) involved in stem cell mobilization and cytotoxic drugs combined with G-CSF suppressed the function of osteoblasts and HSPCs, so we hypothesized that stimulation of the HSPC niche, rather than the stem cell itself, may provide therapeutic benefit for clinical SCT. Here we found that PTH-treated mice showed a significant increase in the absolute number and function of osteoblasts. The level of osteocalcin mRNA in the PTH and $P+G$ groups was significantly higher than that in the CTL or G group $(P<0.01$; Fig. $4 \mathrm{a})$. Consistent with gene expression analysis, the number of CD45 $5^{-}$Ter $119^{-}$ $\mathrm{OPN}^{+}$osteoblasts in the PTH $(2780 \pm 197$ cells/femur $)$ and $\mathrm{P}+\mathrm{G}(2768 \pm 236$ cells/femur $)$ groups was significantly higher than that in the CTL $(1091 \pm 114$ cells/ femur) and $G(954 \pm 87$ cells/femur $)$ groups $(P<0.01$; Fig. 4b). Moreover, the density of trabeculae in the long bones was significantly increased in the PTH and P + G groups (Fig. 4c). PTH treatment also enhanced osteoblast function. Of the two PTH-treated groups, the osteocalcin level in the PTH and P + G groups was $51.89 \pm 5.17 \mathrm{ng} / \mathrm{ml}$ and $36.43 \pm 1.89 \mathrm{ng} / \mathrm{ml}$, respectively, while its level was $30.39 \pm 1.47 \mathrm{ng} / \mathrm{ml}$ in the CTL group and $28.34 \pm 0.65 \mathrm{ng} /$ $\mathrm{ml}$ in the $\mathrm{G}$ group $(P=0.02$; Fig. $4 \mathrm{~d})$.

RANKL can stimulate osteoclasts and elevate the serum TRACP 5b level $[15,16]$. Here we observed that RANKL treatment significantly increased the number of osteoclasts in the trabecula bone ( $\mathrm{P}+\mathrm{R}$ group vs. $\mathrm{PTH}$ group; Fig. 5a). The serum TRACP-5b level was also significantly elevated after RANKL treatment $(12.16 \pm 0.52 \mathrm{U} / \mathrm{l})$ compared with PTH treatment only $(3.87 \pm 1.28 \mathrm{U} / \mathrm{l})(P=$ 0.01; Fig. 5b). These data suggest that PTH may overcome the side effect of cytotoxic chemotherapy on osteoblasts. Moreover, RANKL treatment can efficiently activate osteoclast function.

\section{PTH and RANKL can protect and expand the resident HSC} pool during cytotoxic chemotherapy

Our previous data demonstrated that interrupting the balance of bone remodeling can facilitate HSPC mobilization [9]. In this study, the hematopoietic repopulation activity 


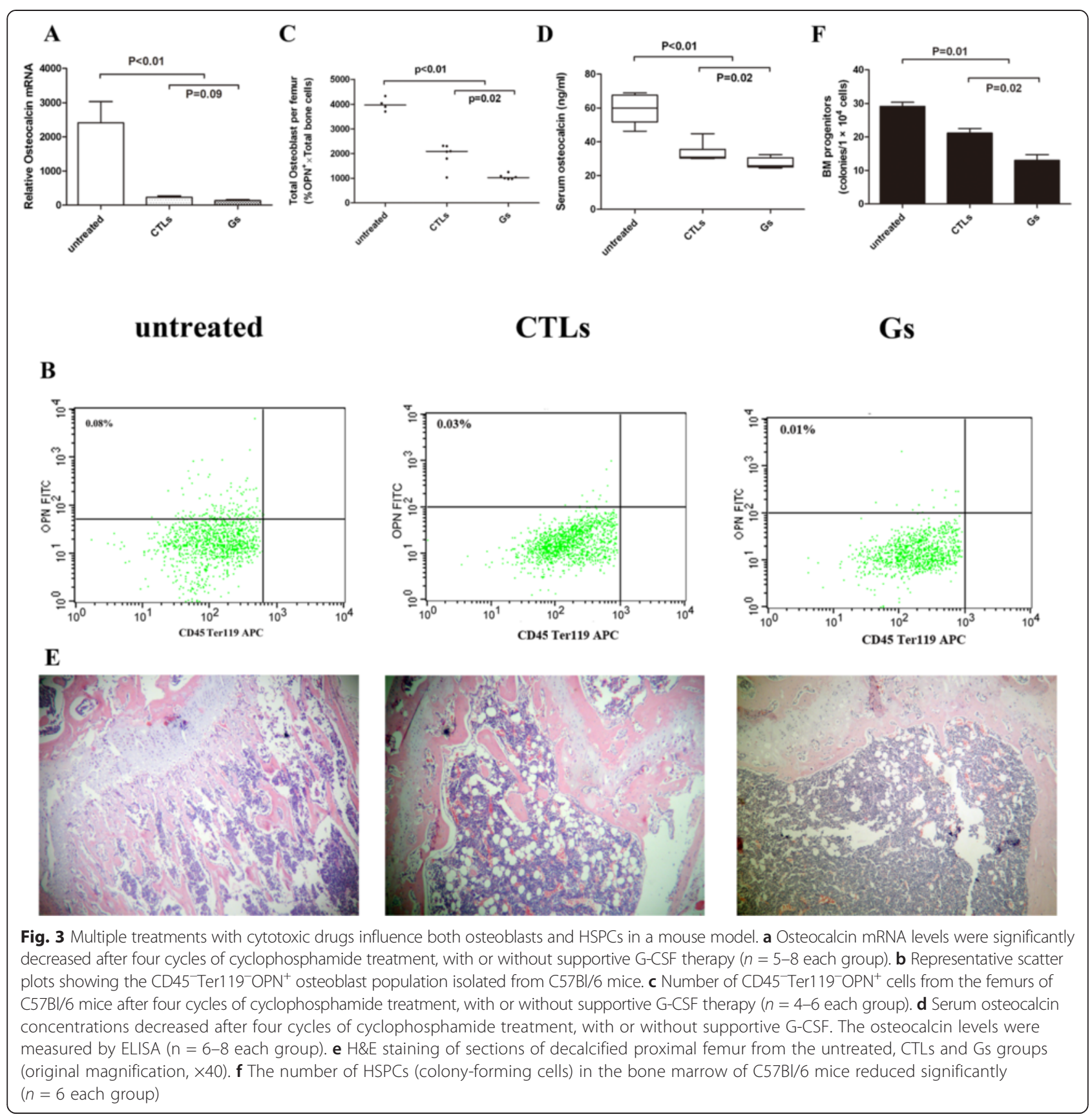

of HSCs and transplanted HSPCs was assessed at 16 weeks after transplantation by flow cytometric analysis of donor-derived (CD 45.2) myeloid, mature B and T cells in the recipients' (CD 45.1) PB. CRA analysis of bone marrow HSPCs showed that there was a significant depletion of the HSC pool in the mice treated with G-CSF (CTL $11.2 \%$ CD $45.2^{+}$chimerism vs. G $4.8 \%$ CD $45.2^{+}$chimerism, $P=0.01$ ) (Fig. 6a). However, treatment with PTH resulted in relative preservation of the HSC pool (G vs. PTH 16.7 \% CD $45.2^{+}$chimerism, $P<0.01$; CTL vs. PTH, $P<0.05$ ) (Fig. 6a). Similarly, analysis of the mobilization of HSCs into the peripheral circulation demonstrated that in mice who did not receive supportive G-CSF therapy during chemotherapy there was mobilization of HSCs into the circulation, while mice who received supportive G-CSF therapy alone showed little mobilization of HSCs into the peripheral circulation (CTL $10.8 \%$ CD $45.2^{+}$chimerism vs. G $3.3 \%$ CD $45.2^{+}$chimerism, $P<0.05$ ) (Fig. $6 \mathrm{~b}$ ). This was partially reversed by the following treatment with PTH (G vs. $\mathrm{P}+\mathrm{G} 19.5 \%$ CD $45.2^{+}$chimerism, $P<0.01$; CTL vs. $P+G, P<0.05$ ) (Fig. $6 \mathrm{~b}$ ). Moreover, recipient mice transplanted with circulation HSPCs from the $\mathrm{P}+\mathrm{R}$ and $\mathrm{P}+\mathrm{R}+\mathrm{G}$ groups showed more robust myeloid and lymphatic cell engraftment than those in the mice 


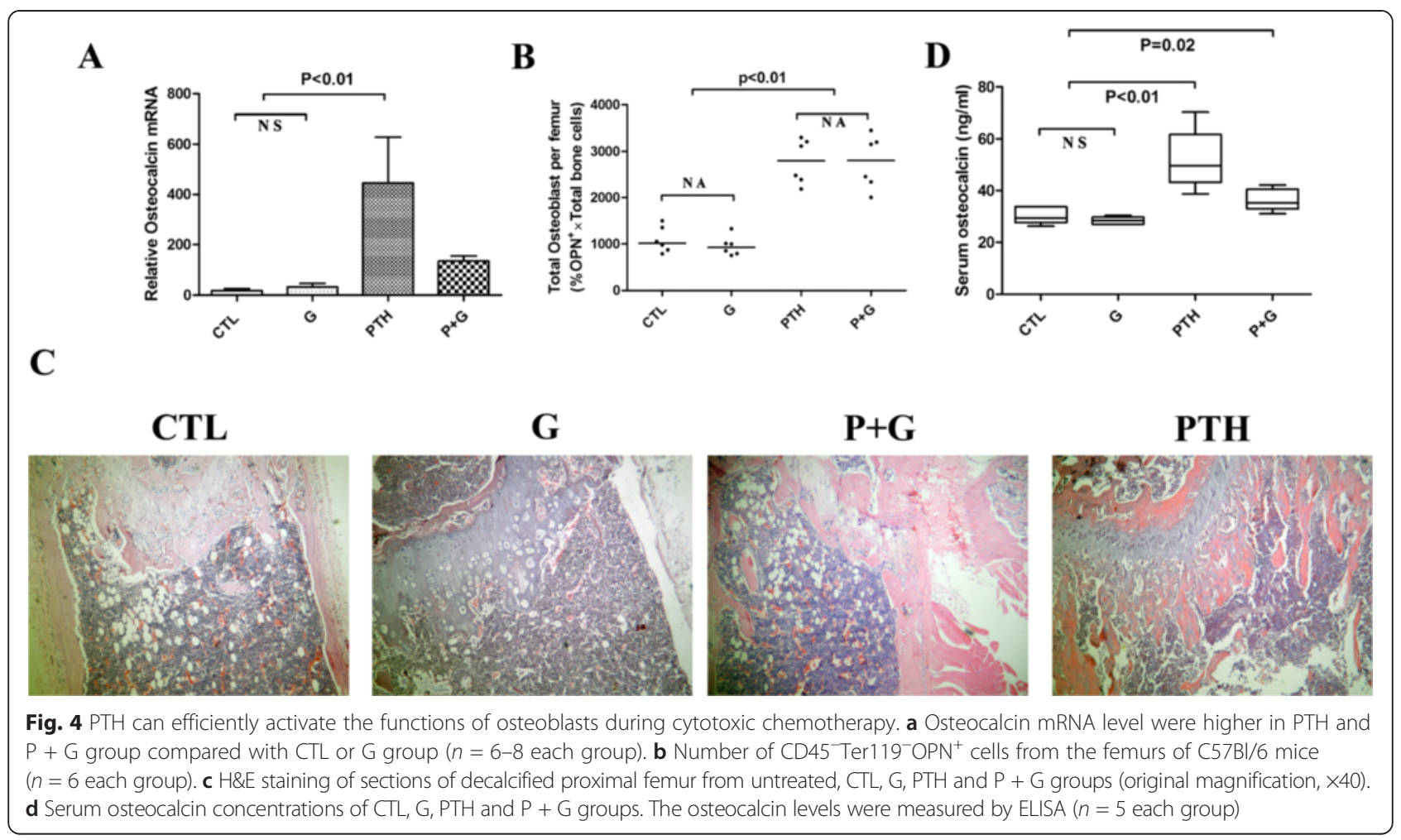

transplanted with HSCs from either the CTL or G group (Fig. 6b). RANKL could mobilize HSC efficiently as well as G-CSF (P + G vs. P + R $16.8 \%$ CD $45.2^{+}$chimerism, $P$ $>0.05$ ) (Fig. 6b). However, RANKL treatment failed to amplify the mobilization of HSCs treated with PTH and G-CSF (P + G vs. P + R + G $17.5 \%$ CD $45.2^{+}$chimerism, $P$ $>0.05$ ) (Fig. 6b). These data indicate that cytotoxic chemotherapy markedly depletes HSCs in bone marrow. Targeting the niche cells can protect and expand the resident HSC pool in the bone marrow during chemotherapy, and then effectively counteract the side effect of G-CSF.

\section{Discussion}

In this study, the activity of bone marrow niche osteoblasts was detected in 10 autograft patients and several clinically relevant model systems. As expected, we found that compared with G-CSF treatment alone, cytotoxic chemotherapy combined with G-CSF treatment showed more severe inhibition of osteoblasts as well as HSPCs. Considering both PTH and RANKL are important regulators for bone remodeling [20-25], they were added to manipulatively interrupt the balance of bone remodeling. Finally, our data show that PTH and RANKL treatment

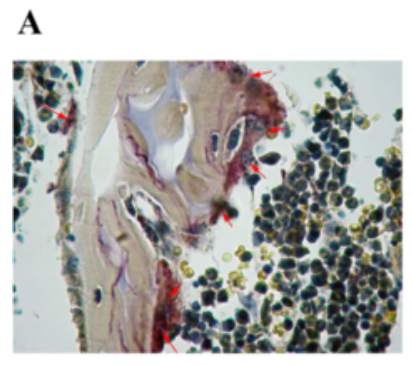

$\mathbf{P}+\mathbf{R}$
B

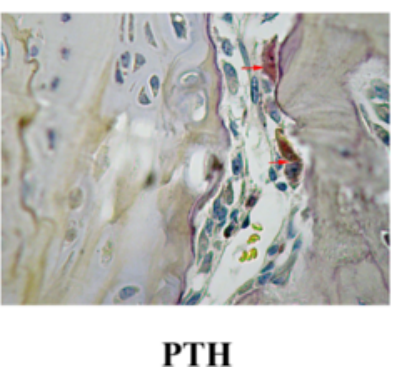

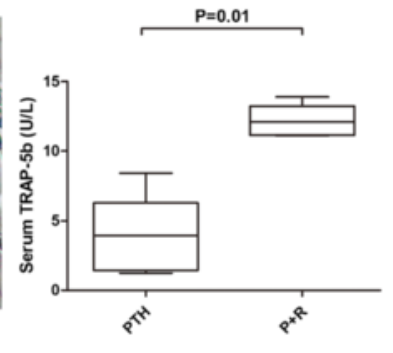

Fig. 5 RANKL can efficiently activate the functions of osteoclasts during cytotoxic chemotherapy. a TRAP staining of mouse femoral metaphysis of PTH and P + R groups. Arrowheads: active TRAP ${ }^{+}$osteoclasts stained in red (original magnification, $\times 400$ ). $\mathbf{b}$ Serum TRAP-5b level also detected by ELISA ( $n=5$ each group). $P+R$ mice injected with PTH and RANKL, PTH parathyroid hormone, TRAP 5b, tartrate-resistant acid phosphatase 5b 

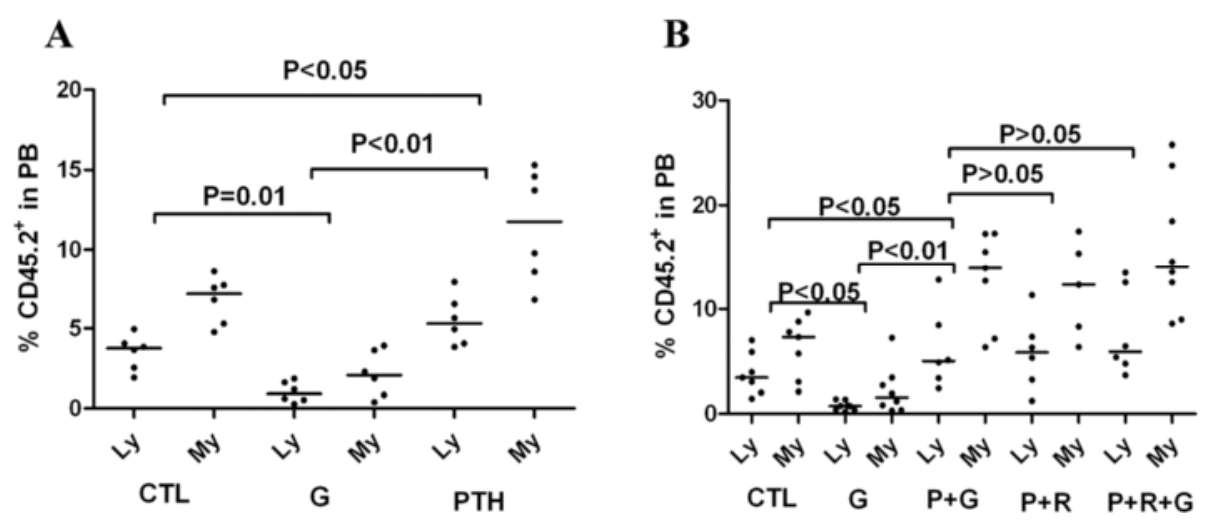

Fig. 6 PTH and RANKL can protect and expand the resident HSC pool during cytotoxic chemotherapy. a Measurement of the HSC content of the bone marrow in the CTL, $\mathrm{G}$ and PTH groups by CRA at 16 weeks ( $n=6$ each group). $\mathbf{b}$ Measurement of the HSC content of the PB of mice in the $C T L, G, P+G, P+R$ and $P+R+G$ groups by CRA at 16 weeks ( $n=6-8$ each group)

can protect and even expand the resident HSC pool during cytotoxic chemotherapy.

HSCs reside within specific bone marrow niches and are anchored by adhesive interactions [26, 27], so repetitive cycles of chemotherapy may damage the niche and disrupt HSC functions such as quiescence, proliferation, and self-renewal [28]. During HSC mobilization in healthy donors, G-CSF significantly increases the number of HSCs in $\mathrm{PB}$, but suppresses the number and the activities of osteoblasts in the meantime $[5,29,30]$. Our previous data have demonstrated the pivotal role of bone remodeling in the above processes, so it is reasonable to manipulate this balance to improve the mobilization efficiency [9]. During autologous SCT, a standard procedure has been applied, which involves multiple rounds of chemotherapy followed by G-CSF-induced mobilization of HSPCs [1]. How about bone remodeling in autologous SCT? Winkler et al. [28] demonstrated that the effects of cyclophosphamide and G-CSF in the metaphyseal spongiosa are similar to those of loss of osteoblasts, transient arrest in bone formation, and reduced CXCL12 expression. In this study, we also observed that the osteoblast niche was impaired by multiple rounds of cytotoxic drug treatment before G-CSF mobilization, in which the serum osteocalcin level obviously declined from $22.19 \pm 1.08 \mathrm{ng} / \mathrm{ml}$ to $16.08 \pm 2.12 \mathrm{ng} / \mathrm{ml}$. We further verified these findings in two clinically relevant mouse models. The number of trabeculae and endosteal osteoblasts and the serum osteocalcin level in the long bones were significantly decreased in cyclophosphamidetreated mice, an effect that was further aggravated by GCSF treatment. These data indicate that osteoblasts are destructed during autologous transplantation, even more severe than that in healthy donors. Moreover, multiple rounds of chemotherapy can significantly deplete the bone marrow HSPC pool, which is in accordance with the published report [5]. Protection of the niche function is therefore more important to ameliorate the poor mobilizers.

Considering the close proximity and importance of osteoblasts to HSPCs, we further demonstrated that both G-CSF and cyclophosphamide suppressed niche-supportive osteoblasts, and thus inhibited the expression of endosteal cytokines and resulted in major impairment of HSC reconstitution potential in the mobilized bone marrow. Based on the above findings, multiple rounds of cytotoxic chemotherapy (particularly when combined with G-CSF) impaired bone marrow function, and also limited the ability of patients to receive multiple rounds of optimal chemotherapy afterwards or limited the ability to obtain suitable stem cell products before a salvage bone marrow transplantation. Strategies to maintain the stem cell number and function in these clinical situations would therefore be desirable. Previous studies have demonstrated that both hematopoiesis and the HSC niche related to the bone remodeling process may be modulated by PTH and RANKL activity [31-33]. Our previous study also showed that, compared with G-CSF mobilization, an increase or decrease of the osteoblast/osteoclast ratio was closely related to the number of HSPCs in PB, suggesting that the imbalance of bone remodeling could facilitate HSPC mobilization [9]. To further verify whether the activation of endosteal niches can improve HSPC transplantation in a poor mobilization model, here we established six mouse models relevant to clinical uses of HSPCs. Our data showed that PTH treatment could increase the absolute number and function of osteoblasts, indicating that PTH may counteract the negative effects of cytotoxic drugs and G-CSF on osteoblasts. Moreover, we demonstrated that PTH treatment could increase the number of stem cells mobilized into $\mathrm{PB}$ and protect bone marrow stem cells from repeated exposure to cytotoxic chemotherapy and G-CSF. Furthermore, RANKL could also mobilize HSPCs to $\mathrm{PB}$, so it may be used as a valuable alternative to G- 
CSF. Our data indicate that targeting the endosteal niche cells is a potential therapeutic approach to enhance stem cell-based therapies.

Our data are also supported by previous studies. Constitutive activation of the PTH receptor in osteoblasts increased the HSC number and activity [34]. Ballen et al. [35] performed a phase I study for PTH and found that 47 $\%$ of patients with hematologic malignancies acquired adequate $\mathrm{CD} 34^{+}$cells with the help of PTH. Moreover, RANKL-stimulated bone-resorbing osteoclasts reduce the stem cell niche components SDF-1, SCF, and OPN in the endosteum and finally trigger HSPC mobilization, so RANKL may be used together with other mobilization agents in an extensive range of clinical HSPC transplantation protocols [12]. In the present study, the CRA showed that recipient mice transplanted with circulation HSPCs from the $P+R$ and $P+R+G$ groups had more robust myeloid and lymphatic cell engraftment than either the CTL or G group. These findings suggest that stimulation of the endosteal bone marrow niche can lead to increased engraftment of the HSC compartment through increased expansion of the stem cell pool.

\section{Conclusions}

Using clinical specimens and clinically relevant models, we demonstrate that manipulation of bone remodeling can increase the efficiency of HSC mobilization and targeting on the HSC niche is a viable and reasonable therapeutic choice in stem cell therapy. More importantly, our findings provide new knowledge for the development and treatment of poor mobilization.

\begin{abstract}
Abbreviations
ACK: Ammonium-chloride-potassium; ALL: Acute lymphoblastic leukemia; APC: Allophycocyanin; CAMS: Chinese Academy of Medical Sciences; CRA: Competitive repopulation assay; DAB: 3,3'-Diaminobenzidine; EDTA: Ethylenediamine tetraacetic acid; ELISA: Enzyme-linked immunosorbent assay; FACS: Fluorescence-activated cell sorting; FITC: Fluorescein isothiocyanate; G-CSF: Granulocyte colony-stimulating factor; H\&E: Hematoxylin and eosin; HSC: Hematopoietic stem cell; HSPC: Hematopoietic stem/progenitor cell; NHL: Non-Hodgkin's lymphoma; PB: Peripheral blood; PBS: Phosphate-buffered saline; PE: Phycoerythrin; PTH: Parathyroid hormone; PUMC: Peking Union of Medical College; RANKL: Receptor activator of nuclear factor kappa-B ligand;
\end{abstract} SCT: Stem cell transplantation; TRACP 5b: Tartrate-resistant acid phosphatase 5b.

\section{Competing interests}

The authors declare that they have no competing interests.

\section{Authors' contributions}

Sidan Li carried out the animal studies, collected the patients' samples, and drafted the manuscript. Dehui Zou and Changhong Li helped collect the patients' samples. Hengxing Meng and Weiwei Sui carried out the flow cytometry. Sizhou Feng and Tao Cheng participated in the design of the study, performed the statistical analysis and helped to revise the manuscript. Qiongli Zhai and Lugui Qiu conceived of the study, participated in its design and coordination, and helped to draft the manuscript. All authors read and approved the final manuscript.

\section{Acknowledgements}

This work was supported by grants from the National Natural Science Foundation of China (No. 81300433, No. 81370632 and No. 81272361), the Beijing Municipal
Natural Science Foundation (No. 7144211), the Specialized Research Fund for the Doctoral Program of Higher Education (No. 20131107120008), the BCH Young Investigator Program (BCHYIPA-2013-06) and Beijing Municipal Administration of Hospitals Clinical Medicine Development of special funding support (No. ZY201404).

\section{Author details}

${ }^{1}$ State Key Laboratory of Experimental Hematology, Institute of Hematology and Hospital of Blood Diseases, Chinese Academy of Medical Sciences and Peking Union of Medical College, 288 Nanjing Road, Tianjin 30020, China. ${ }^{2}$ Beijing Key Laboratory of Pediatric Hematology Oncology, National Key Discipline of Pediatrics, Ministry of Education, Hematology Oncology Center, Beijing Children's Hospital, Capital Medical University, Beijing, China.

${ }^{3}$ Department of Pathology, Tianjin Medical University Cancer Institute and Hospital, National Clinical Research Center for Cancer, Key Laboratory of Cancer Prevention and Therapy, Tianjin 300060, China.

Received: 15 January 2015 Revised: 25 January 2015 Accepted: 19 August 2015 Published online: 15 September 2015

\section{References}

1. To LB, Levesque JP, Herbert KE. How I treat patients who mobilize hematopoietic stem cells poorly. Blood. 2011;118:4530-40.

2. Bensinger W, DiPersio JF, McCarty JM. Improving stem cell mobilization strategies: future directions. Bone Marrow Transplant. 2009;43:181-95.

3. Bonig H, Papayannopoulou T. Mobilization of hematopoietic stem/ progenitor cells: general principles and molecular mechanisms. Methods Mol Biol. 2012;904:1-14.

4. Motabi IH, DiPersio JF. Advances in stem cell mobilization. Blood Rev. 2012;26:267-78.

5. Bonig H, Papayannopoulou T. Hematopoietic stem cell mobilization: updated conceptual renditions. Leukemia. 2013;27:24-31.

6. Piccirillo N, Vacca M, Lanti A, Ipsevich F, Maresca M, Fiorelli E, et al. Poor mobilizer: a retrospective study on proven and predicted incidence according to GITMO criteria. Transfus Apher Sci. 2012;47:217-21.

7. Hoggatt J, Pelus LM. Hematopoietic stem cell mobilization with agents other than G-CSF. Methods Mol Biol. 2012;904:49-67.

8. Mohty M, Ho AD. In and out of the niche: perspectives in mobilization of hematopoietic stem cells. Exp Hematol. 2011;39:723-9.

9. Li S, Zhai Q, Zou D, Meng H, Xie Z, Li C, et al. A pivotal role of bone remodeling in granulocyte colony stimulating factor induced hematopoietic stem/progenitor cells mobilization. J Cell Physiol. 2013;228:1002-9.

10. Jin F, Zhai Q, Qiu L, Meng H, Zou D, Wang Y, et al. Degradation of BM SDF-1 by MMP-9: the role in G-CSF-induced hematopoietic stem/progenitor cell mobilization. Bone Marrow Transplant. 2008:42:581-8.

11. Adams GB, Martin RP, Alley IR, Chabner KT, Cohen KS, Calvi LM, et al. Therapeutic targeting of a stem cell niche. Nat Biotechnol. 2007;25:238-43.

12. Kollet O, Dar A, Shivtiel S, Kalinkovich A, Lapid K, Sztainberg Y, et al. Osteoclasts degrade endosteal components and promote mobilization of hematopoietic progenitor cells. Nat Med. 2006;12:657-64.

13. Huang W, Zou D, Sui W, Wang T, Fu M, Li Z, et al. Clinical outcome of autologous stem cell transplantation as first-line treatment in 30 patients with high risk lymphoblastic lymphoma. Zhonghua Xue Ye Xue Za Zhi. 2014;35:332-6.

14. Liu X, Jiang E, Huang Y, Yang D, Pang A, Wei J, et al. Outcome of autologous hematopoietic stem cell transplantation in combination with imatinib for 16 cases of Philadelphia chromosome positive acute lymphoblastic leukemia. Zhonghua Xue Ye Xue Za Zhi. 2014;35:250-2.

15. Zou DH, Sui WW, Yi SH, An G, Xu Y, Li ZJ, et al. Analysis of the efficacy and prognosis on first-line autologous hematopoietic stem cell transplantation of patients with multiple myeloma. Zhonghua Xue Ye Xue Za Zhi. 2013;34:299-303.

16. Christopher MJ, Link DC. Granulocyte colony-stimulating factor induces osteoblast apoptosis and inhibits osteoblast differentiation. J Bone Miner Res. 2008:23:1765-74.

17. Mayack SR, Wagers AJ. Osteolineage niche cells initiate hematopoietic stem cell mobilization. Blood. 2008;112:519-31. Blood. 2012;119:1793.

18. Weitzmann MN. The role of inflammatory cytokines, the RANKL/OPG axis, and the immunoskeletal interface in physiological bone turnover and osteoporosis. Scientifica (Cairo). 2013;2013:125705. 
19. Feng W, Xia W, Ye Q, Wu W. Osteoclastogenesis and osteoimmunology. Front Biosci (Landmark Ed). 2014;19:758-67.

20. Augustine M, Horwitz MJ. Parathyroid hormone and parathyroid hormonerelated protein analogs as therapies for osteoporosis. Curr Osteoporosis Rep. 2013;11:400-6.

21. Inoue D. Role for PTHrP in bone and cartilage metabolism. Clin Calcium. 2014;24:863-9.

22. Kovacs CS. Bone development and mineral homeostasis in the fetus and neonate: roles of the calciotropic and phosphotropic hormones. Physiol Rev. 2014;94:1143-218.

23. Martin TJ. Historically significant events in the discovery of RANK/RANKL/ OPG. World J Orthop. 2013;4:186-97.

24. Dougall WC, Holen I, Gonzalez SE. Targeting RANKL in metastasis. Bonekey Rep. 2014;3:519.

25. Honma M, Ikebuchi Y, Kariya Y, Suzuki H. Regulatory mechanisms of RANKL presentation to osteoclast precursors. Curr Osteoporos Rep. 2014;12:115-20.

26. Mendelson A, Frenette PS. Hematopoietic stem cell niche maintenance during homeostasis and regeneration. Nat Med. 2014;20:833-46.

27. Riether C, Schurch CM, Ochsenbein AF. Regulation of hematopoietic and leukemic stem cells by the immune system. Cell Death Differ. 2014;4:187-98.

28. Winkler IG, Pettit AR, Raggatt $\amalg$, Jacobsen RN, Forristal $C E$, Barbier $V$, et al. Hematopoietic stem cell mobilizing agents G-CSF, cyclophosphamide or AMD3100 have distinct mechanisms of action on bone marrow HSC niches and bone formation. Leukemia. 2012;26:1594-601.

29. Singh P, Hu P, Hoggatt J, Moh A, Pelus LM. Expansion of bone marrow neutrophils following G-CSF administration in mice results in osteolineage cell apoptosis and mobilization of hematopoietic stem and progenitor cells. Leukemia. 2012;26:2375-83.

30. Semerad CL, Christopher MJ, Liu F, Short B, Simmons PJ, Winkler I, et al. G-CSF potently inhibits osteoblast activity and CXCL12 mRNA expression in the bone marrow. Blood. 2005;106:3020-7.

31. Garrett RW, Emerson SG. The role of parathyroid hormone and insulin-like growth factors in hematopoietic niches: physiology and pharmacology. Mol Cell Endocrinol. 2008;288:6-10.

32. Pacifici R. Osteoimmunology and its implications for transplantation. Am J Transplant. 2013;13:2245-54.

33. Arboleya L, Castaneda S. Osteoimmunology: the study of the relationship between the immune system and bone tissue. Reumatol Clin. 2013;9:303-15.

34. Calvi LM, Adams GB, Weibrecht KW, Weber JM, Olson DP, Knight MC, et al. Osteoblastic cells regulate the haematopoietic stem cell niche. Nature. 2003:425:841-6.

35. Ballen KK, Shpall EJ, Avigan D, Yeap BY, Fisher DC, McDermott K, et al. Phase I trial of parathyroid hormone to facilitate stem cell mobilization. Biol Blood Marrow Transplant. 2007:13:838-43.

\section{Submit your next manuscript to BioMed Central and take full advantage of:}

- Convenient online submission

- Thorough peer review

- No space constraints or color figure charges

- Immediate publication on acceptance

- Inclusion in PubMed, CAS, Scopus and Google Scholar

- Research which is freely available for redistribution 